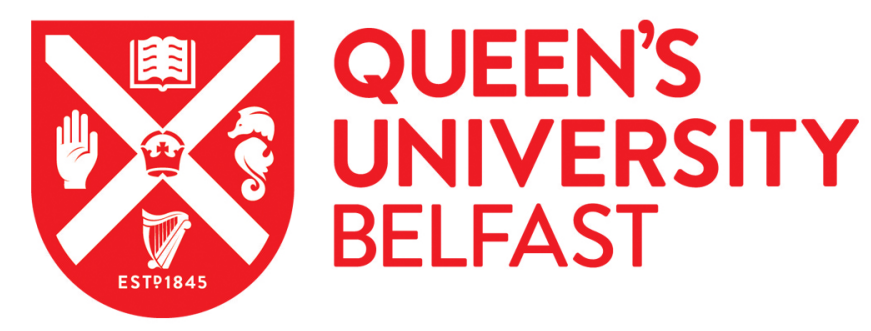

\title{
On-line simulation of 2D resonators with reduced dispersion error using compact implicit finite difference methods
}

Kowalczyk, K., \& Van Walstijn, M. (2007). On-line simulation of 2D resonators with reduced dispersion error using compact implicit finite difference methods. 285-288. Paper presented at 32nd IEEE International Conference on Acoustics, Speech and Signal Processing, Honolulu, United States.

https://doi.org/10.1109/ICASSP.2007.366672

Document Version:

Early version, also known as pre-print

Queen's University Belfast - Research Portal:

Link to publication record in Queen's University Belfast Research Portal

\section{General rights}

Copyright for the publications made accessible via the Queen's University Belfast Research Portal is retained by the author(s) and / or other copyright owners and it is a condition of accessing these publications that users recognise and abide by the legal requirements associated with these rights.

Take down policy

The Research Portal is Queen's institutional repository that provides access to Queen's research output. Every effort has been made to ensure that content in the Research Portal does not infringe any person's rights, or applicable UK laws. If you discover content in the Research Portal that you believe breaches copyright or violates any law, please contact openaccess@qub.ac.uk. 


\title{
ON-LINE SIMULATION OF 2D RESONATORS WITH REDUCED DISPERSION ERROR USING COMPACT IMPLICIT FINITE DIFFERENCE METHODS
}

\author{
Konrad Kowalczyk and Maarten van Walstijn
}

\author{
Sonic Arts Research Centre \\ School of Electronics, Electrical Engineering and Computer Science \\ Queen's University of Belfast, Belfast, Northern Ireland \\ kkowalczyk01@qub.ac.uk, m.vanwalstijn@qub.ac.uk
}

\begin{abstract}
This paper presents a method for on-line simulation of 2D resonators with reduced direction-dependent frequency error. The use of a compact implicit finite difference (FD) technique is proposed to reduce the dispersion error remarkably. In particular, a computationally efficient method that allows solving 2D implicit problems with a set of three-diagonal equations, namely the alternating direction implicit is discussed. Efficient equation factorisation together with optimally matched free parameters allows more accurate simulation for wider frequency ranges. With the use of this technique, the dispersion error is limited to $1.1 \%$ within the bandwidth up to half of the Nyquist frequency. The compact implicit scheme is compared to compact explicit FD schemes in terms of numerical dispersion error, membrane impulse response, and computational cost.
\end{abstract}

Index Terms- Acoustic propagation, acoustic signal processing, finite difference methods, FDTD methods, waveguides

\section{INTRODUCTION}

Simulation of vibrating systems such as membranes and plates requires numerically solving the two-dimensional wave equation, for which finite difference methods can be used [1]. These methods are also suited for three-dimensional room auralisation purposes [2]. In reality, the sound wave propagation speed in such resonators is independent of propagation direction. An unwanted side effect of using numerical finite difference (FD) schemes is that they introduce a directiondependent dispersion error. In the original rectangular twodimensional digital waveguide mesh (DWG), which is mathematically equivalent to a compact explicit FD scheme, this error is particularly severe in axial directions [3]. In order to make the error of the rectangular mesh homogeneous in all directions, interpolation techniques have been introduced [4], which make the dispersion error nearly direction-independent

This research has been supported by the European Social Fund.
[5]. However, a significant frequency-dependent dispersion error remains. In order to compensate for the directionally independent dispersion error, Savioja and Välimäki introduced a frequency warping technique [6], with which the remaining maximum dispersion error of the interpolated digital waveguide mesh (IDWM) is reduced to $1.2 \%$ [7]. However, the main disadvantage of the frequency warping technique is that pre- and post-processing has to be executed off-line, which makes this method unsuitable for on-line applications [6]. An on-line frequency warping technique has been treated in [8] for a triangular mesh, but this approach brings about the necessity of increasing the sampling frequency and low-pass filtering of the output signal.

The aim of this paper is to propose the use of an FD method with significantly reduced frequency error without much increase in computational cost, which would be suitable for on-line simulations. Consequently, such an FD method would be applicable to interactive applications.

\section{COMPACT IMPLICIT FINITE DIFFERENCE SCHEMES}

Many higher-order accuracy FD schemes are difficult to design mainly due to ensuring stability near boundaries and low operation count. In particular, 'higher-order large star systems' rely on looking at neighbouring nodes more distant from an update point; therefore these systems are inconvenient due to complicated update formulae near boundaries [9].

In order to reduce the numerical error, the use of a compact implicit method is proposed, as it achieves the highest order of accuracy on the smallest mesh system [9]. The compact implicit approximation to the wave equation relies on updating the node data based on both the neighbouring points in time and space domain, as shown in Figure 1(c). This approach is similar to space interpolation in the interpolated digital waveguide mesh, but in this case all the nearest neighbours in time domain are also included in the update formula. Consequently, a higher accuracy can be achieved for a wider frequency range, relative to sampling frequency. For rectilin- 


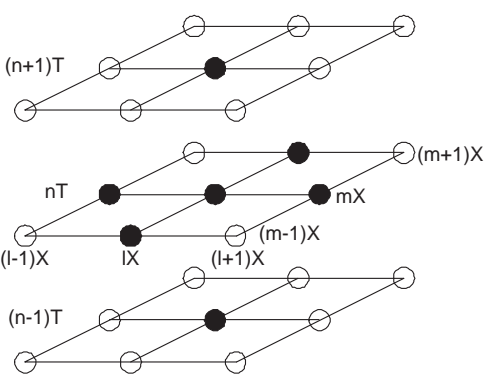

(a) Standard rectilinear stencil

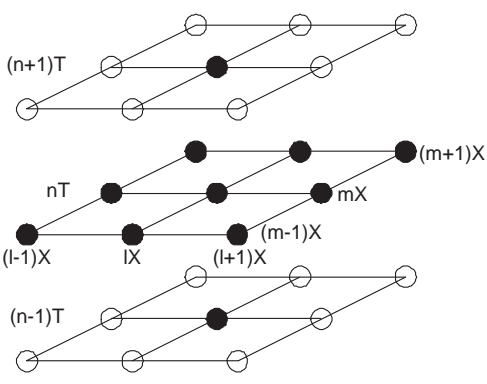

(b) Interpolated rectilinear stencil

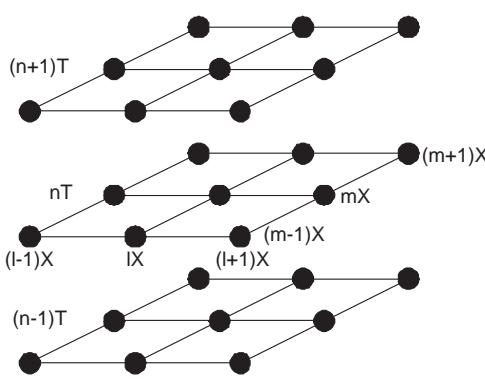

(c) Compact implicit FD stencil

Fig. 1. The mesh structure for: (a) a standard rectilinear scheme, (b) an interpolated rectilinear scheme and (c) a compact implicit finite difference scheme. Black dots denote the mesh nodes taken into account in update formulae.

ear meshes, a general equation for the compact implicit FD scheme approximating the wave equation can be formulated as follows [10]

$$
\begin{aligned}
{\left[1+a\left(\delta_{x}^{2}+\delta_{y}^{2}\right)+a^{2} \delta_{x}^{2} \delta_{y}^{2}\right] \delta_{t}^{2} u_{l, m}^{n}=} & \lambda^{2}\left[\left(\delta_{x}^{2}+\delta_{y}^{2}\right)\right. \\
& \left.+b \delta_{x}^{2} \delta_{y}^{2}\right] u_{l, m}^{n}
\end{aligned}
$$

where $a$ and $b$ are free parameters, $\lambda$ is the Courant number determined by the sampling frequency, $\delta_{t}^{2}, \delta_{x}^{2}$ and $\delta_{y}^{2}$ are standard difference operators approximating second-order derivatives in time and space, respectively, $u_{l, m}^{n}$ is the update variable, $l$ and $m$ denote spatial indexes, and $n$ denotes a time index for neighbouring points.

Example values of free parameters are given in Table 1. For $a=0$, an explicit scheme results. The standard rectilinear scheme, which is equivalent to the digital waveguide mesh, is obtained when both free parameters are set to zero, which leads to the stencil illustrated in Figure 1(a). The stencil in Figure 1(b) results with nonzero values of $b$; this can be obtained by superimposing two standard rectilinear schemes, one of which is rotated by 45 degrees [11]. A nearly frequencyindependent dispersion error can be achieved by setting $b=$ $\frac{1}{6}$, which effectively gives the nearly isotropic scheme described by Trefethen [11]. The interpolated digital waveguide mesh developed by Savioja and Välimäki [4] uses the same stencil, but the coefficients of their difference equation are calculated using an optimisation method; this is effectively equivalent to setting $b=0.1879$.

With regard to the compact implicit FD scheme, adjusting both scheme's free parameters may minimise the problem of the directional dependence of the numerical dispersion [12]. An interesting set of parameters has been proposed by Fairweather and Mitchell [13] as presented in Table 1, for which fourth-order accuracy in both time and space domain is obtained [9]. In comparison, compact explicit schemes can be at the most second-order accurate in both time and space domain. For example, this is the case for the interpolated digital waveguide mesh.

\begin{tabular}{|c|c|c|c|c|}
\hline Par & $\begin{array}{c}\text { Standard } \\
\text { Rectilinear }\end{array}$ & $\begin{array}{c}\text { Interpol. } \\
\text { Rectilinear }\end{array}$ & $\begin{array}{c}\text { Interpol. } \\
\text { DWG }\end{array}$ & $\begin{array}{c}\text { Implicit } \\
\text { Fair\&Mitch }\end{array}$ \\
\hline \hline $\mathrm{a}$ & 0 & 0 & 0 & $\frac{1-\lambda^{2}}{12}$ \\
\hline $\mathrm{b}$ & 0 & $\frac{1}{6}$ & 0.1879 & $\frac{1}{6}$ \\
\hline
\end{tabular}

Table 1. Free parameters for compact finite difference schemes.

\section{ALTERNATING DIRECTION IMPLICIT METHOD}

Multi-dimensional compact implicit methods approximating the wave equation require solving a difference equation at the advanced time level. To address this issue, alternating direction implicit (ADI) methods have been introduced [14], which rely on solving a set of three-diagonal equations. The problem of inverting a matrix is then reduced to a succession of many one-dimensional problems by factorising the scheme [9]. A computationally efficient splitting has been proposed in [10]

$$
\begin{aligned}
& \left(1+a \delta_{x}^{2}\right) u_{l, m}^{n+1 *}=\frac{\lambda^{2}}{a}\left[-1+(a-b) \delta_{y}^{2}\right] u_{l, m}^{n}, \\
& \left(1+a \delta_{y}^{2}\right) \delta_{t}^{2} u_{l, m}^{n}=u_{l, m}^{n+1 *}+\frac{\lambda^{2}}{a}\left(1+b \delta_{y}^{2}\right) u_{l, m}^{n}
\end{aligned}
$$

where $u_{l, m}^{n}$ is the update variable and $u_{l, m}^{n+1 *}$ is an intermediate value. The implementation of an ADI method in twodimensional case consists of two following stages. Firstly, the intermediate values are computed row by row in horizontal direction according to equation (2). Next, the intermediate values are used in computations in vertical direction according to equation (3). In order to preserve the global accuracy, the intermediate boundary values should be obtained explicitly from equation (3). Writing out the standard difference operators in equations 2 and 3 yields the full difference equations

$$
\begin{array}{r}
a\left(u_{l+1, m}^{n+1 *}-c u_{l, m}^{n+1 *}+u_{l-1, m}^{n+1 *}\right)=d\left(u_{l, m}^{n}-e u_{l, m}^{n}+u_{l, m}^{n}\right) \\
a\left(u_{l+1, m}^{n+1}-c u_{l, m}^{n+1}+u_{l-1, m}^{n+1}\right)=u_{l, m}^{n+1 *}+f\left(u_{l, m+1}^{n}+u_{l, m-1}^{n}\right) \\
+g u_{l, m}^{n}-a\left(u_{l+1, m}^{n-1}-c u_{l, m}^{n-1}+u_{l-1, m}^{n-1}\right),
\end{array}
$$




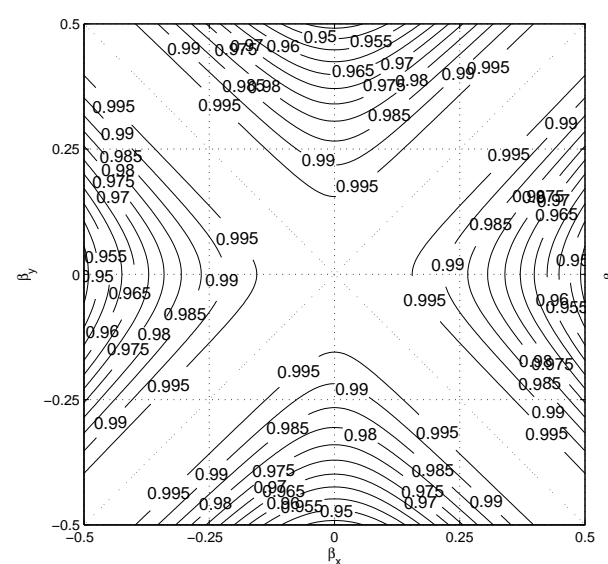

(a) Standard rectilinear mesh

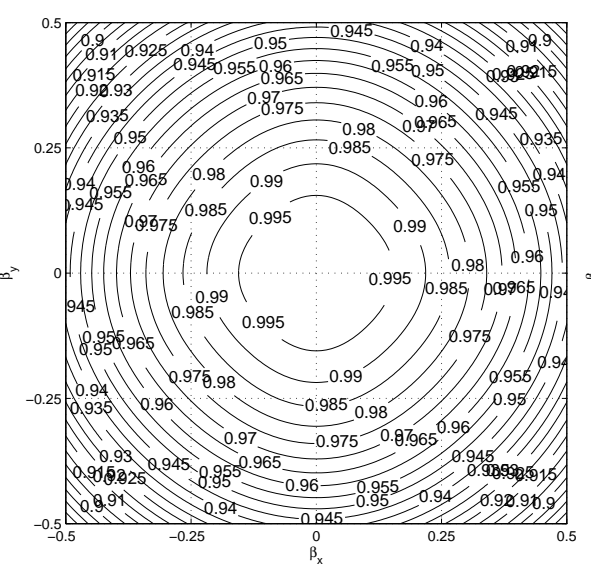

(b) Interpolated digital waveguide mesh

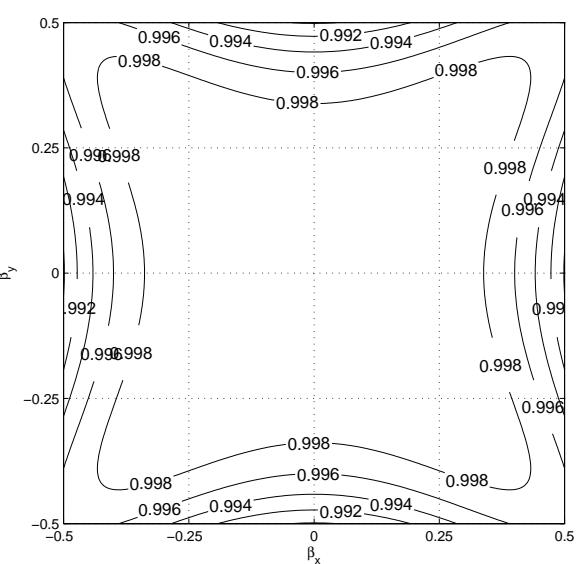

(c) Compact implicit FD mesh

Fig. 2. Relative phase velocity plots for: (a) the standard rectilinear mesh, (b) the interpolated digital waveguide mesh, and (c) the compact implicit FD scheme with optimum free parameters, as a function of the normalised wave numbers in $x$ and $y$ directions.

where the respective coefficients are as follows

$$
\begin{aligned}
& c=2-\frac{1}{a}, \quad d=\frac{\lambda^{2}(a-b)}{a}, \quad e=\left(2+\frac{1}{a-b}\right), \\
& f=2 a+\frac{\lambda^{2} b}{a}, \quad g=2-4 a+\frac{\lambda^{2}}{a}(1-2 b) .
\end{aligned}
$$

For fast implementation of the matrix inversion, there exists an efficient algorithm to inverse the three-diagonal system of linear equations that arise in implicit finite difference schemes, namely, the Thomas algorithm [15].

\section{PERFORMANCE COMPARISON}

\subsection{Numerical dispersion error}

As a measure of dispersion error, the numerical phase velocity relative to the correct velocity is used, as defined in [12]. The comparison of the relative phase velocity for the standard rectilinear scheme, the interpolated digital waveguide mesh and the compact implicit scheme with optimised parameters is presented in Figure 2. The standard rectilinear scheme suffers from a directionally dependent dispersion error with a maximum of $5.5 \%$ in axial directions at half the Nyquist frequency (which corresponds to $\beta=0.5$ ). Figure 2(c) shows that the dispersion error for the compact implicit FD scheme does not exceed $1.1 \%$, which is significantly better when compared to the interpolated digital waveguide mesh, for which the maximum dispersion error amounts to $12 \%$, as illustrated in Figure 2(b). The dispersion of the IDWM can be reduced to a similarly small amount using off-line frequency warping techniques.

\subsection{Membrane response}

In this section, a numerical simulation example of a rectangular membrane with clamped edges is presented to compare the performance of both the compact FD explicit and implicit methods. The simulated membrane consists of a $15 \times 15$ mesh structure with outer nodes assigned a constant value of zero. Following [16], the mesh was excited with an impulse filtered with a FIR filer of a transfer function $H(z)=1-z^{-2}$. An impulse was injected in one corner node $(2,2)$ and the receiver was located in the opposite corner $(14,14)$ of the membrane. The magnitude of the impulse responses for the interpolated digital waveguide mesh and the compact implicit FD scheme are compared to theoretical modal frequencies in Figure 3(a) and (b), respectively. With the interpolated digital waveguide mesh, the numerical simulation brings about systematic shifts in modal frequencies, particularly at high frequencies. In comparison, the resonance frequencies resulting with the compact implicit FD scheme using the parameters presented in Table 1 match well with analytical values for a significantly wider frequency range.

\subsection{Computational cost}

Table 2 shows, for each of the three schemes, the number of arithmetic operations and memory access operations for each junction at each time step. The necessity of storing values from two previous time steps concerns all three algorithms, and thus the number of memory locations is the same in that regard. In comparison to explicit schemes, the increase in the number of multiplications for compact implicit scheme is offset by the lower number of memory access operations per grid point.

In order to achieve a $1.1 \%$ maximum dispersion error up to half Nyquist, as with the compact implicit FD mesh, the in- 


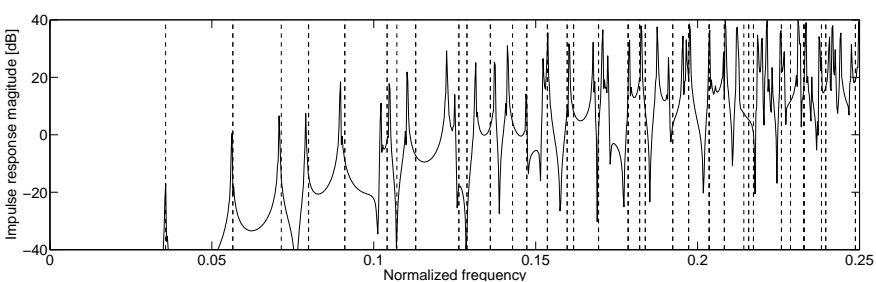

(a) Interpolated digital waveguide mesh

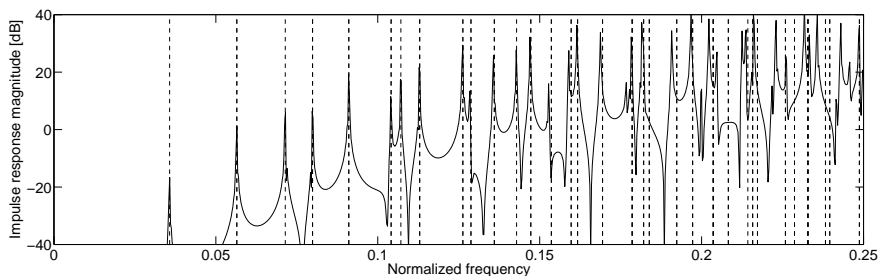

(b) Compact implicit FD mesh

Fig. 3. Magnitude of the membrane impulse response for: (a) the interpolated digital waveguide mesh without frequency warping, and (b) the compact implicit FD mesh with optimum parameter choice. Dashed lines denote the ideal mode frequencies of the membrane.

\begin{tabular}{|c|c|c|c|}
\hline & Sum/Sub & Mult/Div & Mem Acc \\
\hline \hline Standard Rectilinear & 5 & 2 & 6 \\
\hline Interpolated DWM & 9 & 3 & 10 \\
\hline Compact ADI & 14 & 16 & 7 \\
\hline
\end{tabular}

Table 2. Arithmetic operations and memory access operation count.

terpolated digital waveguide mesh would require a three times higher sample rate, which would result in a nine times denser grid and a three times the number of samples to be computed. Consequently, the number of arithmetic operations would increase twenty seven times and nine times more memory locations would be needed. A more efficient approach would be to double the sample rate in a standard rectilinear simulation, which would result in a four times denser grid and twice the number of samples. Hence the numerical operations count would increase eight times, and four times more memory locations would be necessary. Therefore, the compact implicit FD technique proves to be efficient in terms of computational resources required for on-line simulations.

\section{CONCLUSION}

The compact implicit FD method with optimum parameter choice allows remarkable reduction of the dispersion error. The operation count of compact explicit FD schemes is lower, but in order to achieve the same maximum dispersion error up to half Nyquist, these schemes would have to be run with at least twice the sample rate, which would make them computationally more expensive. While the interpolated digital waveguide mesh requires off-line processing to reduce the dispersion error, the compact implicit scheme offers an efficient method for on-line applications.

\section{REFERENCES}

[1] S. Bilbao, "Finite difference schemes for plate synthesis," Proc. Int. Computer Music Conf., May 2005, Madrid, Spain.

[2] D. Bootteldooren, "Finite-difference time domain simulation of low-frequency room acoustic problems," J. Acoustical Society America, vol. 98, no. 6, pp. 3392-3308, 1995.

[3] J.O. Smith III, "Physical modeling using digital waveguides," Computer Music Journal, vol. 16, no. 4, pp. 74-91, 1992.

[4] L. Savioja and V. Välimäki, "Reduction of the dispersion error in the triangular digital waveguide mesh using frequency warping," IEEE Signal Processing Letters, vol. 6, pp. 58-60, March 1999.

[5] L. Savioja and V. Välimäki, "Improved discrete-time modeling of multi-dimentional wave propagation using the interpolated digital waveguide mesh," Proc. IEEE Int. Conf. Acoustics, Speech and Signal Processing, vol. 2, no. 5, pp. 973-976, March 1999, Phoenix, AZ.

[6] L. Savioja and V. Välimäki, "Reducing the dispersion error in the digital waveguide mesh using interpolation and frequency warping techniques," IEEE Trans. Speech and Audio Processing, vol. 8, March 2000.

[7] L. Savioja and V. Välimäki, "Multiwarping for enhancing the frequency accuracy of digital waveguide mesh simulations," IEEE Signal Processing Letters, vol. 8, no. 5, pp. 134-136, May 2001.

[8] F. Fontana and D. Rocchesso, "Online correction of dispersion error in 2D waveguide meshes," Proc. Int. Computer Music Conf., pp. 78-81, August 2000, Berlin, Germany.

[9] M. Ciment and S. Leventhal, "Higher order compact implicit schemes for the wave equation," Mathematics of Computation, vol. 29, pp. 985-994, October 1975.

[10] A.R. Gourlay and A.R. Mitchell, "A classification of split methods for hyperbolic equations in several space dimentions," SIAM Journal on Numerical Analysis, vol. 6, no. 1, pp. 62-71, March 1969.

[11] L. N. Trefethen, "Group velocity in finite difference schemes," Society for Industrial and Applied Mathematics (SIAM) Review, vol. 24, no. 2, pp. 113-136, April 1982.

[12] S. Bilbao, "Parameterized finite difference schemes for the wave equation," Numerical Methods for Partial Differential Equations, vol. 20, no. 3, pp. 463-480, 2004.

[13] G. Fairweather and A.R. Mitchell, "A high accuracy alternating direction method for the wave equation," J. Inst. Math. Appl., vol. 1, May 1965.

[14] J. Douglas, "Alternating direction methods for three space variables," Numer. Math., vol. 4, pp. 41-63, 1962.

[15] J.C. Strikwerda, Finite difference schemes and partial differential equations, Wadsworth \& Brooks, Pacific Grove, CA, 1989.

[16] M. Karjalainen and C. Erkut, "Digital waveguides vs. finite difference schemes: Equivalence and mixed modeling," EURASIP J. Applied Signal Process., , no. 7, pp. 978-989, June 2004. 